\title{
Epidemiologische Forschung \\ Deutsches Zentrum für Diabetesforschung
}

Matthias Schulze', Annette Peters ${ }^{2}$

1 Abteilung Molekulare Epidemiologie, Deutsches Institut für Ernährungsforschung Potsdam-Rehbrücke

${ }^{2}$ Institute of Epidemiology II, Helmholtz Zentrum München - Deutsches Zentrum für Gesundheit und Umwelt

Die epidemiologische Forschung im Deutschen Zentrum für Diabetesforschung DZD stützt sich auf bereits existierende, große Kohortenstudien, die in den letzten Jahren einen Schwerpunkt auf die Erforschung der Störungen des Metabolismus und die Entwicklung des Diabetes gelegt haben. Diese Studien werden federführend von dem Deutschen Institut für Ernährungsforschung (DIfE) in der Region Potsdam und vom Helmholtz Zentrum München (HMGU) in der Region Augsburg durchgeführt. Beide DZD-Zentren unterhalten gut etablierte Kooperationen mit anderen DZD-Partnern, insbesondere zwischen DIfE und der Universität Tübingen sowie zwischen der HMGU und dem Deutschen Diabetes Zentrum in Düsseldorf. Im Folgenden beschreiben wir die beiden Kohortenstudien und stellen neue Ergebnisse aus den beiden Studien vor. Im Ausblick beschreiben wir die neuen Projekte im Rahmen des DZD.
Abb. 1 Diabetesnachbeobachtung in der EPIC-PotsdamStudie.
Die epidemiologische Forschung im Deutschen Zentrum für Diabetesforschung DZD stützt sich auf bereits existierende, große Kohortenstudien, die in den letzten Jahren einen Schwerpunkt auf die Erforschung der Störungen des Metabolismus und die Entwicklung des Diabetes gelegt haben.

\section{Beschreibung der EPIC-Kohorte}

Die European Prospective Investigation into Cancer and Nutrition (EPIC)-Potsdam-Studie ist eine prospektive Kohortenstudie mit 27548 Teilnehmern. Frauen im Alter von 35 bis 64 Jahren und Männer im Alter von 40 bis 64 Jahren wurden zwischen 1994 und 1998 für die Studie gewonnen [1]. Potenzielle Studienteilnehmer wurden dabei nach einem Zufallsverfahren aus den Einwohnermelderegistern von Potsdam und der ländlichen Umgebung gezogen und anschließend angeschrieben. Die Teilnehmerrate betrug $23 \%$. Informationen zum Lebensstil und zu Ernährungsgewohnheiten wurden durch selbstausfüllbare und computerlesbare Fragebögen erfasst. In einem Studienzentrum wurden PCgestützte Interviews und verschiedene Messungen (Anthropometrie, Blutdruck, Knochendichte) sowie eine Blutabnahme durchgeführt. Im Rahmen der Nachbeobachtung werden sowohl Daten über das Auftreten neuer Erkrankungen als auch über Risikofaktoren gesammelt. Der

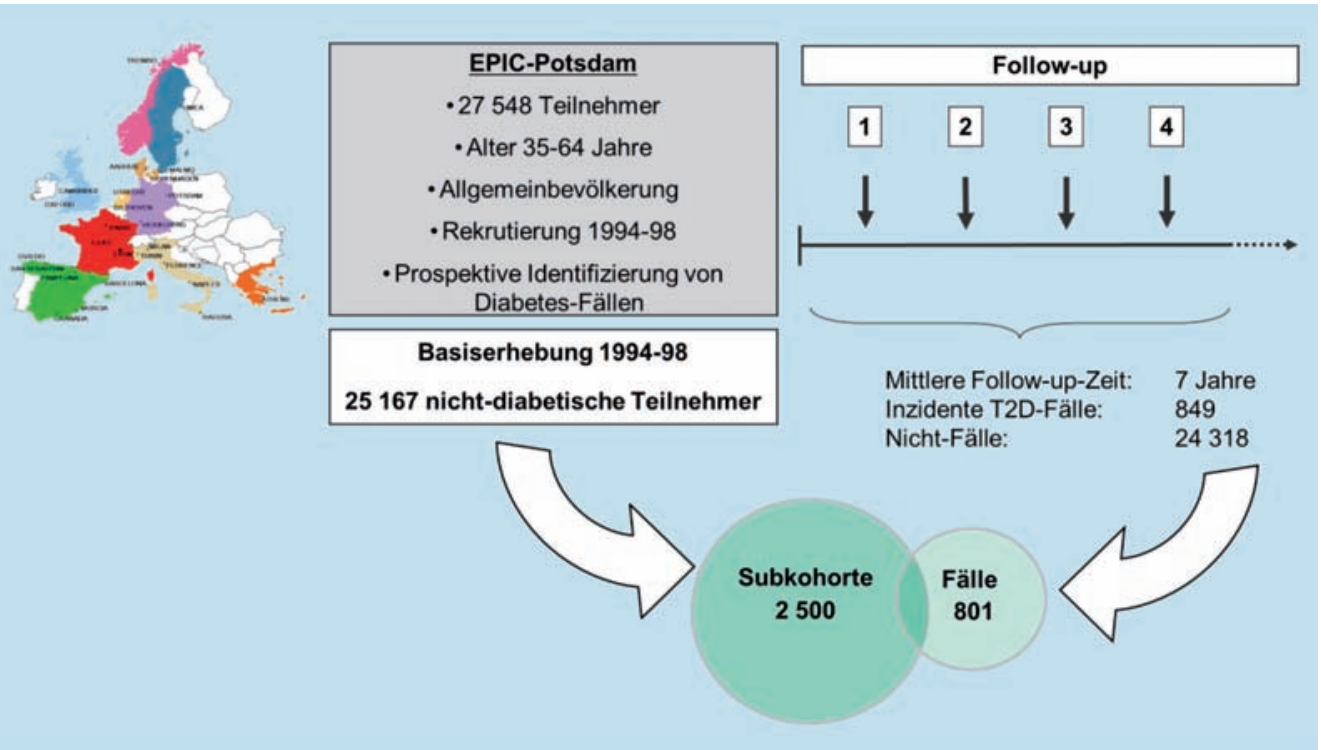


Kontakt zu den Studienteilnehmern erfolgt dabei mit Fragebögen. Selbstangaben der Studienteilnehmer zu Diabeteserkrankungen werden durch Kontaktierung des diagnostizierenden Arztes hinsichtlich Typ-2-Diabetes verifiziert. Nur bestätigte Fälle werden in die statistischen Analysen eingebracht. Bis 2005 wurden in der Kohorte 849 Neuerkrankungen an Typ-2-Diabetes registriert (Abb. 1).

\section{Der Deutsche Diabetes-Risiko-Test ${ }^{\circledR}$}

Für gezielte Maßnahmen zur Prävention des Typ-2-Diabetes ist es notwendig, Personen mit erhöhtem Erkrankungsrisiko zu identifizieren. Dazu wurde anhand der Daten der EPIC-Potsdam-Studie ein Risikoscore entwickelt, der es erlaubt, anhand von anthropometrischen Merkmalen, Informationen zum Lebensstil und der Ernährung eine präzise Voraussage der Erkrankungswahrscheinlichkeit vorzunehmen [2]. Der Score wurde in der EPIC-Heidelberg-Studie validiert. Der Deutsche Diabetes-Risiko-Test ${ }^{\circledR}$ ist als interaktives Webtool zugänglich und liegt auch in einer vereinfachten Papierversion vor (www. dife.de, Abb. 2). Der Test wird von der Deutschen Diabetes-Gesellschaft als Screeninginstrument zur Bestimmung des Risikos einer zukünftigen und zur Identifizierung einer bereits vorhandenen Diabeteserkrankung im Rahmen der aktuellen Praxisleitlinien empfohlen [3]. Im Vergleich $\mathrm{zu}$ anderen verfügbaren Risikotests lässt der Deutsche Diabetes-Risiko-Test ${ }^{\circledR}$ eine relativ akkurate Risikobestimmung zu [4]. Auch haben Personen mit erhöhtem Diabetesrisiko ein erhöhtes kardiovaskuläres und Mortalitätsrisiko [5]. Dennoch konnte anhand der Daten der EPICPotsdam-Studie festgestellt werden, dass sich die Vorhersagegüte des Tests insbesondere durch die Bestimmung der Plasmaglukose und des $\mathrm{HbA}_{1 \mathrm{c}}{ }^{-}$ Wertes verbessert [6]. Untersuchungen ergaben allerdings, dass die Informationen zu 20 mit einem erhöhten Typ-2-Diabetesrisiko assoziierten Genvarianten für die Vorhersage einer zukünftigen Diabeteserkrankung unbrauchbar sind. Auch in Zukunft wird die Evaluation neuer biochemischer Parameter und genetischer Marker zur Risikoprädiktion ein Schwerpunkt am DIfE sein. Daneben ist die Validierung der Prädiktionsmodelle in der KORA-Studie im Forschungsprogramm des DZD verankert.

\section{Lebensstil und Risiko für Diabetes in EPIC-Potsdam}

Übergewicht als wichtigster Risikofaktor für Diabetes wird üblicherweise anhand des BodyMass-Index (BMI) erfasst. Als Alternative zum BMI wurde vor kurzem der Body-Adiposity-Index (BAI) vorgeschlagen, der neben der Körperlänge den Hüftumfang einer Person berücksichtigt. Die Aussagekraft des BAI zur Bestimmung

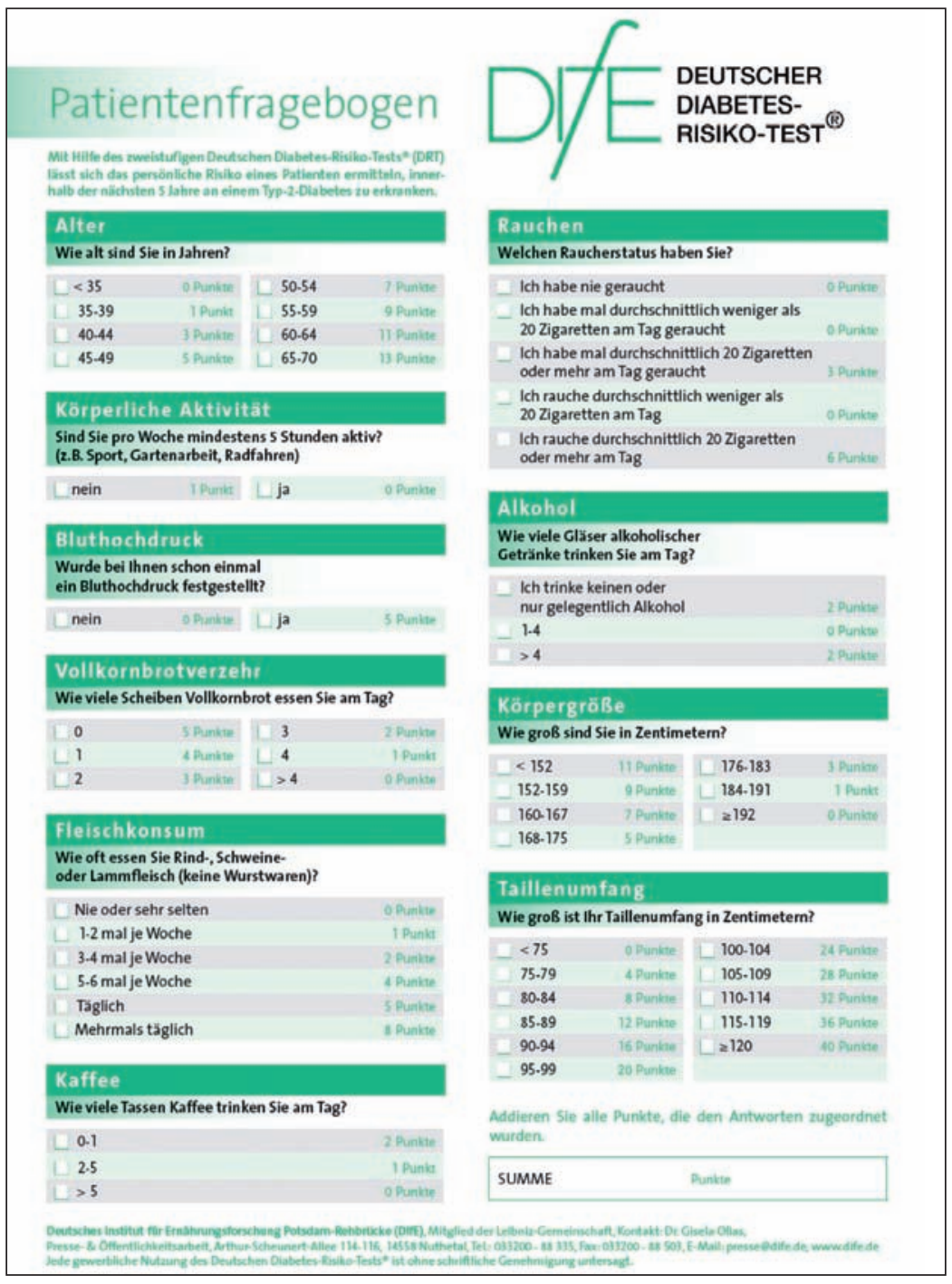

Abb. 2 Der Deutsche Diabetes-Risiko-Test ${ }^{\circledR}$.

des Körperfetts und des Diabetesrisikos im Vergleich zu Hüftumfangs- und TaillenumfangsMessungen sowie der Aussagekraft des BMI wurde in einem Gemeinschaftsprojekt des DZD untersucht [7]. Hierfür wurden Daten von Studienteilnehmern des „Tübinger Lebensstil-Interventions-Programms" (TULIP), der EPIC-Potsdam-Studie sowie der KORA-Studie genutzt. Als Goldstandard für den Vergleich der verschiedenen Messmethoden dienten Magnetresonanztomografie-Messungen, die den Körperfettanteil sehr genau bestimmen und im Rahmen der Tübinger Studie durchgeführt worden waren. In der Studie konnte gezeigt werden, dass der BAI dem altbekannten BMI bei der Einschätzung des prozentualen Körperfettanteils nicht überlegen ist und der BMI sogar in einer engeren Beziehung zur Körperfettverteilung steht als der BAI. Insbesondere bei männlichen Studienteilnehmern war die Einschätzung des prozentualen Körperfettanteils mithilfe des BAI ungenau. Auch bei der Bestimmung des Diabetesrisikos der Studienteilnehmer war der BMI dem BAI überlegen, 
Abb. 3 Übersicht über die KORA-Studie.

\section{$1985 \rightarrow$ KORA Myocardial Infarction Register $\rightarrow 2009$}

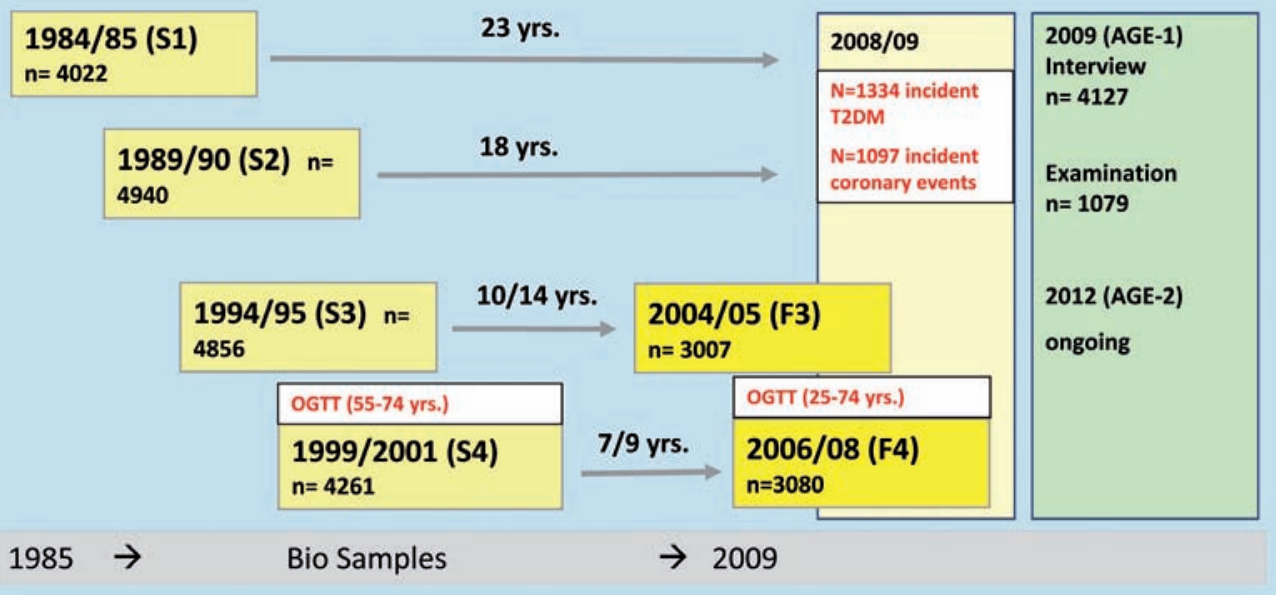

allerdings besaßen beide Indizes hinsichtlich der Diabetesrisiko-Einschätzung eine geringere Aussagekraft als der gemessene Taillenumfang. Viele Ernährungsfaktoren werden im Zusammenhang mit Typ-2-Diabetes diskutiert. Die epidemiologische Forschung am DIfE befasst sich hier mit einzelnen Nährstoffen, dem Zusammenspiel von Nährstoffen durch die Auswahl von Lebensmitteln und mit Ernährungsmustern [8, 9]. Wer niemals geraucht hat, nicht massiv übergewichtig ist, pro Woche mehr als dreieinhalb Stunden körperlich aktiv ist und sich gesund ernährt, hat im Vergleich zu einer Person, die sich gegenteilig verhält, ein um $93 \%$ vermindertes Risiko, an Typ-2-Diabetes zu erkranken [10].

Biomarkerforschung in der EPIC-Potsdam-Studie Biomarker spiegeln das biologisch relevante Profil an der Schnittstelle zwischen der Zufuhr von Nährstoffen und Stoffwechselprozessen wider. Das Fettsäureprofil der Erythrozytenmembranen stellte sich in der EPIC-Potsdam-Studie als starker Prädiktor des Diabetesrisikos heraus. Hier waren insbesondere Mengenverhältnisse von Fettsäuren, die Aktivitäten der im PUFAStoffwechsel (Polyunsaturated Fatty Acids) wichtigen Desaturasen widerspiegeln, für das Diabetesrisiko relevant [11]. Diese Beobachtung konnte auch durch Genotypinformationen bestätigt werden. Neben Biomarkern der Ernährung werden in der EPIC-Potsdam-Studie metabolische und genetische Marker hinsichtlich ihrer Bedeutung für das Diabetesrisiko untersucht. So konnte u. a. in Zusammenarbeit mit der Universität Tübingen beobachtet werden, dass höhere Plasmaspiegel des Hepatokins Fetuin-A, für welches in der Tübinger Studie ein wichtiger Zusammenhang mit der intrahepatischen Fettakkumulation identifiziert wurde, mit einem erhöhten Diabetesrisiko in EPIC-Potsdam assozi- iert sind [12]. Die Identifizierung und Evaluation von neuen Risikomarkern wird die Abteilung in Kooperation mit anderen Abteilungen des DIfE und den Partnern des Deutschen Zentrums für Diabetesforschung e.V. durchführen, um so von den verschiedenen Humanstudien mit unterschiedlichem Phänotypisierungsgrad und von der Vernetzung mit experimentellen Forschergruppen optimal zu profitieren.

\section{Beschreibung der KORA-Kohorte}

Die KORA-Kohorte baut sich aus den Teilnehmern von unabhängigen, populationsbasierten Querschnittsuntersuchungen in der Stadt Augsburg und den Landkreisen Augsburg und Aichach-Friedberg auf (Abb. 3) [13]. Insgesamt wurden in der Region 4 unabhängige bevölkerungsbasierte Surveys durchgeführt. Die ersten 3 im Rahmen des WHO-MONICA (MONItoring trends and determinants in CArdiovascular disease)-Projektes in den Jahren 1984/85 (S1), 1989/90 (S2) und 1994/95 (S3). Nach Abschluss des MONICA-Projektes wurde vom Helmholtz Zentrum München die Forschungsaktivitäten in der Region unter dem Namen KORA (Kooperative Gesundheitsforschung in der Region Augsburg) weitergeführt und 1999/2001 (S4) der vierte Survey durchgeführt. Das Alter zur Basisuntersuchung war 25-64 Jahre (S1) beziehungsweise 74 Jahre (S2-S4). Die Stichprobe wurde stratifiziert nach Stadt-/Landbevölkerung sowie Geschlecht und 10-Jahres-Altersgruppen aus den Einwohnermeldeämtern gezogen. Einschlusskriterium in die Studie war die deutsche Staatsangehörigkeit und erster Wohnsitz in der Studienregion. Die Studienbeteiligung lag damals zwischen 79\% in S1 und 67\% in S4.

Zur weiteren Beobachtung werden alle Probanden in regelmäßigen Abständen angeschrieben und gebeten, Veränderungen des Gesundheits- 
zustands mitzuteilen. Dadurch ist es möglich, Neuerkrankungen („Inzidenzen“) für wichtige Krankheitsbilder zu identifizieren. Ferner wird erhoben, ob und woran Probanden gestorben sind. 8000 Probanden nahmen bisher zusätzlich an Wiederholungsuntersuchungen im Studienzentrum teil. Die Nachuntersuchungen erfolgten für die Surveys S3 und S4, wobei sich im Laufe der Zeit sowohl das Methodenspektrum bei den Untersuchungselementen als auch bei den Biomaterialien erweiterte. Die letzte vollständige Adressrecherche wurde 2008/09 durchgeführt, gefolgt von einer schriftlichen Befragung aller Überlebenden. Dabei wurden 3108 Todesfälle registriert und bei der Befragung eine Teilnehmerrate von $80 \%$ erreicht.

\section{Häufigkeit von Glukosestoffwechselstörung} und Diabetes in der Modellregion Augsburg Basierend auf der KORA-F4-Studie konnte gezeigt wurden, dass im Alter zwischen 35 und 59 Jahren die Hälfte aller Fälle mit Diabetes (Gesamtanteil 4\%) nicht diagnostiziert waren und zusätzliche $11 \%$ einen gestörten Glukosestoffwechsel hatten [14]. Bei den älteren Personen haben im Zeitraum von 7 Jahren 10,5\% einen Diabetes entwickelt, was einer Inzidenz von 15,5 Fällen pro 1000 Personenjahren entspricht [15]. Ein Risikoscore konnte aus den Daten abgeleitet werden, der auf Angaben beruht, die dem Allgemeinarzt zur Verfügung stehen. Bei älteren Personen lässt sich das Risiko für Typ-2-Diabetes gut durch Angaben zu Alter, Geschlecht, BMI, Diabetes der Eltern, Bluthochdruck, Rauchen, Nüchternglukose, Harnsäure sowie $\mathrm{HbA}_{1 \mathrm{c}}$ vorhersagen [16].

Eine Reihe von Lebensstilfaktoren ist mit einem Diabetesrisiko verbunden, was im Rahmen der KORA-Studie bestätigt werden konnte. Dazu gehören Übergewicht und insbesondere abdominales Übergewicht, verminderte körperliche Aktivität, Bluthochdruck und das Alleineleben. Insbesondere bei Personen mit gestörtem Glukosemetabolismus ist sowohl aktives als auch passives Zigarettenrauchen mit einem erhöhten Diabetesrisiko verbunden. Bei Frauen wurde beobachtet, dass ein spätes Auftreten der Menarche das Risiko im Laufe des Lebens reduziert [17]. Hiermit ergibt sich ein Ansatz, einer Untergruppe von Frauen gezielt Interventionen, insbesondere bezüglich einer Gewichtsreduzierung, anzubieten.

\section{Entzündungsmarker und Typ-2-Diabetes}

Die Untersuchung von klassischen und neuen Risikofaktoren für Typ-2-Diabetes wurde in den vergangenen Jahren in KORA als Schwerpunkt etabliert. Biomarker für Entzündungsreaktionen, der endothelialen Dysfunktion und des Fettgewebes standen im Mittelpunkt der im vor- herigen Absatz beschriebenen Fall-KohortenStudie. Es konnte gezeigt werden, dass Marker der endothelialen Dysfunktion, wie lösliches E-Selektin oder intrazelluläres Adhäsionsmolekül-1, und Entzündungsmarker, wie C-reaktives Protein, Zytokine und Chemokine, das Risiko einen inzidenten Diabetes zu entwickeln anzeigen (Tab. 1). Bei einigen Markern war die Vorhersage insbesondere bei Frauen sehr gut. Im Gegensatz zu den koronaren Herzerkrankungen haben Personen mit einem veränderten Leptin zu Adiponektion Verhältnis ein verändertes Diabetesrisiko.

Neue Untersuchungen an Teilnehmern der KORAStudie haben gezeigt, dass Menschen mit guter Vitamin-D-Versorgung ein geringeres Risiko haben, an Typ-2-Diabetes zu erkranken [18]. Personen mit zu geringen Konzentrationen von Vitamin D im Blut haben ein erhöhtes Risiko. Dieser Effekt könnte unter anderem auf die entzündungsmindernde Wirkung von Vitamin D zurückzuführen sein. Das Ergebnis der am Helmholtz Zentrum München in Zusammenarbeit mit Dr. Christian Herder vom Deutschen Diabetes Zentrum Düsseldorf und Prof. Dr. Wolfgang König von der Universität Ulm durchgeführten Studie könnte direkte Auswirkungen auf die Prävention der Volkskrankheit haben.

\section{Neue Ansätze}

\section{für die Diabetes-Epidemiologie}

Das Ziel des Deutschen Diabetes Zentrum ist es, die Vorhersage für die Entwicklung von Typ-2Diabetes zu verbessern. Auf diesem Gebiet gibt es viele neue Entwicklungen, die allerdings für Deutschland bewertet werden müssen, um daraus einheitliche Empfehlungen entwickeln zu können.

Dabei stehen einige Faktoren im Fokus, die aus der Sicht der Bevölkerung große Bedeutung haben und dem Bereich Public-Health-Forschung zu gerechnet werden. Dabei sind die Rolle der Adipositas und der Ernährung sehr wichtige

$\begin{array}{ll}\text { Tab. } 1 & \text { Qualitative Zusammenfassung der } \\ \text { Ergebnisse der KORA-Studie zu Entzündungsmarkern, } \\ \text { endothelialer Dysfunktion und Vitamin D. }\end{array}$


Epidemiological Research - German Center for Diabetes Research

The epidemiological research in the German Center for Diabetes Research (GCDR) is based on already existing, large cohort studies that in the past few years have placed their focus on research into metabolic disorders and the development of diabetes. These studies were carried out under the direction of the German Institute for Nutritional Research (GINR) in the Potsdam region and the Helmholtz Center Munich (HCM) in the Augsburg region. Both diabetes centers are involved in well-established cooperative projects with other GCDR partners, worthy of special mention are those between GINR and the University of Tübingen and between HCM and the German Diabetes Center in Düsseldorf. In the present article we describe the two cohort studies and present new results from the two trials. We also prospectively mention new projects within the framework of the GCDR.

Key words

Diabetes risk score - Cohort studies - Waist circumference - EPIC - KORA
Schwerpunkte. Ein neues sich entwickelndes Feld ist die Untersuchung von Umweltfaktoren, die möglicherweise durch strengere Umweltstandards beeinflusst werden können, wie zum Beispiel die Luftqualität in städtischen Ballungsgebieten.

Es wird erwartet, dass im Rahmen des DZD ein besseres Verständnis der Pathomechanismen entwickelt werden wird und personalisierte Ansätze zur Prävention und Behandlung entwickelt werden. Daher stellt die Bewertung und der Einsatz neuer Krankheitsmarker in bevölkerungsbezogenen Studien eine völlig neue Dimension dar [19].

Sowohl für die Erforschung neuer Ansätze der Verbesserung der individuellen als auch der Gesundheit auf der Bevölkerungsebene, sind die beiden großen epidemiologischen Studien ideal geeignet.

\section{Literatur}

1 Boeing H, Wahrendorf J, Becker N. EPIC-GermanyA source for studies into diet and risk of chronic diseases. European Investigation into Cancer and Nutrition. Ann Nutr Metab 1999; 43: 195-204

2 Schulze MB, Hoffmann K, Boeing $\mathrm{H}$ et al. An accurate risk score based on anthropometric, dietary, and lifestyle factors to predict the development of type 2 diabetes. Diabetes Care 2007; 30: 510-515

3 Kerner W, Brückel J. Definition, Klassifikation und Diagnostik des Diabetes mellitus. Diabetologie 2010; 5 (Suppl. 02): S109-S112

4 Buijsse B, Simmons RK, Griffin SJ, Schulze MB. Risk assessment tools for identifying individuals at risk of developing type 2 diabetes. Epidemiol Rev 2011; 33: 46-62

5 Heidemann C, Boeing $\mathrm{H}$, Pischon T et al. Association of a diabetes risk score with risk of myocardial infarction, stroke, specific types of cancer, and mortality: a prospective study in the European Prospective Investigation into Cancer and Nutrition (EPIC)-Potsdam cohort. Eur J Epidemiol 2009; 24: 281-288

6 Schulze MB, Weikert C, Pischon T et al. Use of multiple metabolic and genetic markers to improve the prediction of type 2 diabetes: the EPIC-Potsdam Study. Diabetes Care 2009; 32: 2116-2119

7 Schulze MB, Thorand B, Fritsche A et al. Body adiposity index, body fat content and incidence of type
2 diabetes. Diabetologia 2012; 55: Feb 17. [Epub ahead of print]

8 Schulze MB, Schulz M, Heidemann C et al. Fiber and magnesium intake and incidence of type 2 diabetes: a prospective study and meta-analysis. Arch Intern Med 2007; 167: 956-965

9 Heidemann C, Hoffmann K, Spranger J et al. A dietary pattern protective against type 2 diabetes in the European Prospective Investigation into Cancer and Nutrition (EPIC)-Potsdam Study cohort. Diabetologia 2005; 48: 1126-1134

10 Ford ES, Bergmann MM, Kroger J et al. Healthy living is the best revenge: findings from the European Prospective Investigation Into Cancer and NutritionPotsdam study. Arch Intern Med 2009; 169: 13551362

11 Kroger J, Zietemann V, Enzenbach C et al. Erythrocyte membrane phospholipid fatty acids, desaturase activity, and dietary fatty acids in relation to risk of type 2 diabetes in the European Prospective Investigation into Cancer and Nutrition (EPIC)-Potsdam Study. Am J Clin Nutr 2011; 93: 127-142

12 Stefan N, Fritsche A, Weikert C et al. Plasma fetuin-A levels and the risk of type 2 diabetes. Diabetes 2008; 57: 2762-2767

13 Holle R, Happich M, Lowel H, Wichmann HE. KORAa research platform for population based health research. Gesundheitswesen 2005; 67 (Suppl 1): S19-S25

14 Meisinger C, Strassburger K, Heier M et al. Prevalence of undiagnosed diabetes and impaired glucose regulation in 35-59-year-old individuals in Southern Germany: the KORA F4 Study. Diabet Med 2010; 27: 360-362

15 Rathmann W, Strassburger K, Heier M et al. Incidence of Type 2 diabetes in the elderly German population and the effect of clinical and lifestyle risk factors: KORA S4/F4 cohort study. Diabet Med 2009; 26: 1212-1219

16 Rathmann W, Kowall B, Heier M et al. Prediction models for incident Type 2 diabetes mellitusin the older population: KORA S4/F4 cohort study. Diabet Med 2010; 27: 1116-1123

17 Stockl D, Doring A, Peters A, Thorand B, Heier M, Huth C, Stockl H, Rathmann W, Kowall B, Meisinger C. Age at menarche is associated with prediabetes and diabetes in women (aged 32-81 years) from the general population: the KORA F4 Study. Diabetologia 2011; 54 [online first]

18 Thorand B, Zierer A, Huth C et al. Effect of serum 25-hydroxyvitamin $D$ on risk for type 2 diabetes may be partially mediated by subclinical inflammation: results from the MONICA/KORA Augsburg study. Diabetes Care 2011; 34: 2320-2322

19 Suhre K, Shin SY, Petersen AK et al. Human metabolic individuality in biomedical and pharmaceutical research. Nature 2011; 477: 54-60

\section{Korrespondenz}

Prof. Dr. Matthias Schulze

Abteilung Molekulare Epidemiologie

Deutsches Institut für Ernährungsforschung

Potsdam-Rehbrücke

Arthur-Scheunert-Allee 114-116

14558 Nuthetal

E-Mail: mschulze@dife.de

\section{Autorenerklärung}

Der Autor erklärt, dass für diesen Artikel kein

Interessenkonflikt besteht. 